\title{
La relevancia de las marcas de carne de vacuno para los consumidores. Un análisis interregional
}

\author{
H. Resano ${ }^{1,3, *}$ y A.I. Sanjuán ${ }^{2,3}$ \\ 1 Facultad de Veterinaria, Departamento de Ciencias Agrarias y del Medio Natural, Universidad de \\ Zaragoza, España \\ 2 Centro de Investigación y Tecnología Agroalimentaria de Aragón, Departamento de Economía Agro- \\ alimentaria y de los Recursos Naturales, Zaragoza, España \\ 3 Instituto Agroalimentario de Aragón (IA2), Universidad de Zaragoza-CITA, España
}

\section{Resumen}

A pesar de que la utilización de la marca permite ayudar a diferenciar el producto y a conseguir un mayor grado de lealtad entre los consumidores, la carne de vacuno se vende habitualmente sin marca. Este trabajo investiga el nivel de conocimiento y de compra de la carne de vacuno con distintos tipos de marca y su relación con la calidad percibida y las características de los consumidores. Conocer el papel que ocupa la marca en la mente del consumidor y en la decisión de compra puede ser especialmente relevante para crear marcas exitosas que estén dirigidas a segmentos específicos. El estudio se ha realizado en dos regiones españolas (Aragón y Cataluña) y en dos francesas (Midi-Pyrénées y Languedoc-Roussillon) con distintos hábitos de consumo entre Septiembre 2010 y Abril 2011. En total se obtuvieron 1,222 encuestas. Los principales métodos de análisis que se han aplicado son la segmentación en dos pasos, las medidas de asociación bivariantes y los contrastes no paramétricos. Se han identificado dos segmentos, el denominado "Conocedor y comprador de carne de vacuno con marca" y el "Menos conocedor y comprador de carne de vacuno con marca". Estos segmentos se caracterizan por presentar unas percepciones de calidad distintivas y un perfil específico. No se ha obtenido un único segmento para todas las regiones, a pesar de que existen algunas coincidencias entre ellas, lo que puede ser de especial interés para discernir si sería aconsejable que se desarrollase una política de marketing distinta en función de la región.

Palabras clave: Análisis cluster, marcas, carne de vacuno, consumidores.

\section{Abstract \\ The role of beef brands for consumers. A cross-regional analysis}

Beef has been traditionally sold as an unbranded product. Nevertheless, the use of a brand would help to differentiate the product and achieve a higher degree of loyalty among consumers. The paper investigates the level of recognition and purchase of different types of branded beef, and relates that with quality perceptions and consumer traits. A better understanding of the role that the brand occupies in consumers' awareness and purchase decisions and its relationship with quality perceptions and personal traits may be relevant to create successful brands targeted to specific segments. Thus, a survey was carried out in two Spanish regions (Aragón and Cataluña) and two French regions (Midi-Pyrénées and Languedoc-Roussillon), with different beef consumption habits, between September 2010 and April 2011,

\footnotetext{
* Autor para correspondencia: mhresano@unizar.es http://doi.org/10.12706/itea.2017.017
} 
obtaining a total sample of 1222 consumers. Main methods of analysis included two-step clustering, bivariate association measures, and non-parametric tests. Cluster analysis revealed the presence of two market segments, tentatively named 'Connoisseur and purchaser of branded beef' and 'Novice and non-purchaser of branded beef'. These segments were characterized by distinctive quality perceptions and a specific socio-demographic and consumption habits profile. Not a common cross-regional segment was found although some coincidences existed. This result can be especially useful for beef producers and distributors to ascertain if they should develop a different marketing policy depending on the region.

Keywords: Cluster analysis, brands, beef, consumers.

\section{Introducción}

La mayoría de la carne que se vende para el consumo en el hogar en los países europeos no está etiquetada con ninguna marca, sobre todo la que se vende en fresco, o sin procesar (Grunert et al., 2004). Por este motivo, los consumidores pueden no disponer de información suficiente para formar sus expectativas sobre la calidad del producto basándose en los indicadores de calidad que estén disponibles en el momento de la compra. Según la teoría de la demanda de Lancaster (1966), la calidad de un bien depende de la de los atributos que lo componen. Estos atributos se denominan de búsqueda, experiencia o confianza, en función de si se pueden evaluar antes de la compra (como el color de la carne), después (su sabor) (Nelson, 1970), o ni lo uno ni lo otro (el sistema de producción) (Darby y Kerni, 1973), respectivamente. En los dos últimos tipos de atributos, y sobre todo en el de confianza se genera un problema de información asimétrica, dado que el productor y/o el vendedor pueden disponer de información sobre el producto que el consumidor no tiene. Sin embargo, estos agentes sí que pueden producir señales para trasmitir esta información al consumidor por ejemplo a través de la utilización de un tipo de marca. La certificación por parte de un tercer agente también permite resolver el problema de asimetría o de información incompleta generada por los atributos de confianza (Compés, 2002), transformándolos en atributos de bús- queda (Sánchez et al., 2007), como puede ser el caso de la alimentación del animal. Los consumidores comparan sus expectativas con la calidad experimentada al consumir el producto para valorar si el producto les satisface o no (Oliver, 1993). La satisfacción con el producto es un elemento crucial desde el punto de vista de los productores y distribuidores para lograr la fidelización del consumidor.

La inherente variación natural que puede experimentar la carne de vacuno complica el proceso de percepción de la calidad e implica que el consumidor puede no estar totalmente seguro de obtener la calidad esperada, existiendo la posibilidad de que sus expectativas no se confirmen en el momento del consumo (Bredahl, 2003). La información proporcionada por la marca facilita la elección entre carnes con diferencias escasamente apreciables según su apariencia visual. Además, la marca puede actuar como un indicador de la calidad más efectivo cuando los consumidores perciben la existencia de un cierto riesgo asociado a la compra del producto (Brunsø et al., 2002). Por este motivo, y dado el elevado número de escándalos alimentarios relacionados con la carne de vacuno que se han producido en los últimos años, como es el caso del fraude de sustitución descubierto en 2013 en varios países europeos de hamburguesas etiquetadas como de carne de vacuno que en realidad eran de caballo (Espinoza et al., 2015) o un año después el de los "kebabs" de carne de vacuno en España e Italia que estaban elaborados fundamentalmente de pollo 
y pavo, respectivamente (BEUC, 2015), no resulta sorprendente comprobar que los consumidores empleen las marcas de carne de vacuno como un elemento adicional para garantizar la seguridad del producto (Sánchez et al., 2001; Banovic et al., 2010).

La compra de carne para el consumo en el hogar se realiza con cierta asiduidad, y la utilización de la marca como indicador de calidad aunque no logra eliminar totalmente la incertidumbre a la que se enfrenta cualquier consumidor que no sea experto en el producto, sí que permite simplificar y aumentar la eficiencia en la compra, ya que aúna gran cantidad de información garantizando una cierta consistencia en el producto elegido (Kotler et al., 2006). Sin embargo, no todas las marcas influyen sobre el proceso de decisión de compra con la misma intensidad, su grado de influencia dependerá del nivel de conocimiento ${ }^{1}$ y credibilidad de la marca, así como del patrocinador o el titular de esa marca. También depende del propio consumidor (Brunsø et al., 2002), y de su nivel de implicación y experiencia con la marca. Por lo que sería interesante clasificar a los consumidores en función de su familiaridad con las marcas para conocer si existen diferencias en la información considerada como relevante en el momento de la compra que tengan consecuencias en la calidad percibida por los distintos consumidores. Su grado de influencia puede estar condicionado también por factores culturales (Brunsø et al., 2002), y por lo tanto, sería relevante conocer si existen o no diferencias interregionales en los consumidores de carne de vacuno. A pesar de que la influencia que ejerce la familiaridad con carne de vacuno sobre la utilización de las marcas como señales de calidad se ha investigado con anterioridad en otros estudios (Bredahl, 2003), ésta es la primera aplicación ${ }^{2}$ cuyo objetivo principal es analizar el efecto de la familiaridad con la marca desde una perspectiva interregional. No obstante, se investiga a su vez si existe una asociación entre la familiaridad con la carne de vacuno y con la marca. Además, se diferencia entre el impacto que presentan distintos tipos de marca como indicadores de calidad en el momento de la compra. Asimismo, se investiga si los consumidores con mayor nivel de conocimiento y compra de carne de vacuno con marca utilizan otros atributos como señales adicionales de calidad. La necesidad de estudiar las preferencias de los consumidores desde una perspectiva regional ha estado presente en la literatura que versa sobre el marketing a lo largo de las últimas décadas (Mittal et al., 2004). A pesar de que el estudio del comportamiento de compra del consumidor en distintos países se ha realizado con mayor profusión, el análisis interregional ofrece ventajas notables respecto a la comparación entre países (Thelen et al., 2006; Mittal et al., 2004). En este sentido, la adopción de un enfoque interregional permite no sólo captar la heterogeneidad existente dentro de cada país, sino también mitigar la inexactitud que se produce cuando se considera una región como representativa del total de la población del país. En el ámbito del marketing agroalimentario, existen relevantes estudios que han demostrado la presencia de diferencias culturales en la percepción de la calidad en la carne (Bernués et al., 2003

1. Según Kotler et al. (2006), las marcas que se reconocen después de sugerirlas y no las que se expresan de manera espontánea ocupan una posición en nuestra mente más influyente en el proceso de decisión de compra.

2. El presente estudio utiliza como punto de partida un trabajo preliminar y exploratorio realizado por Resano et al. (2011) sobre una sub-muestra de consumidores. En el presente trabajo, se ha ampliado la muestra, el abanico de herramientas de análisis y se profundiza en la interpretación de los resultados en el contexto de una revisión de la literatura más extensa. 
a, b), aunque fundamentalmente entre países. En particular, el estudio se ha desarrollado en el marco de un proyecto europeo en dos regiones españolas (Aragón y Cataluña) y dos regiones francesas (Midi-Pyrénées y Languedoc-Roussillon) situadas a ambos lados de los Pirineos (ver Figura 1). La producción de carne de vacuno se considera una actividad relevante para la economía de las cuatro regiones que son objeto de estudio en este artículo (Sanjuán et al., 2012; Sans y Sanjuán-López, 2015), favoreciendo notablemente el desarrollo rural de las zonas próximas a la frontera entre Francia y España. Estas áreas gozan de un notable prestigio para la pro- ducción de carne de vacuno, lo que ha supuesto el reconocimiento a través de las marcas de garantía (Ternera del Valle de Broto, Vedella de Girona, etc...), y en algunos casos incluso han obtenido el reconocimiento por parte de la Comisión Europea mediante la Indicación Geográfica Protegida ${ }^{3}$ (IGP), como ocurre con la Ternera de los Pirineos Catalanes, o está en trámites de su obtención (Carne de Vacuno Montañas de Aragón). Sin embargo, a pesar del elevado desarrollo en los últimos años de la carne de vacuno con este tipo de certificación la penetración en el mercado español y francés es todavía pequeña (Sans et al., 2008).

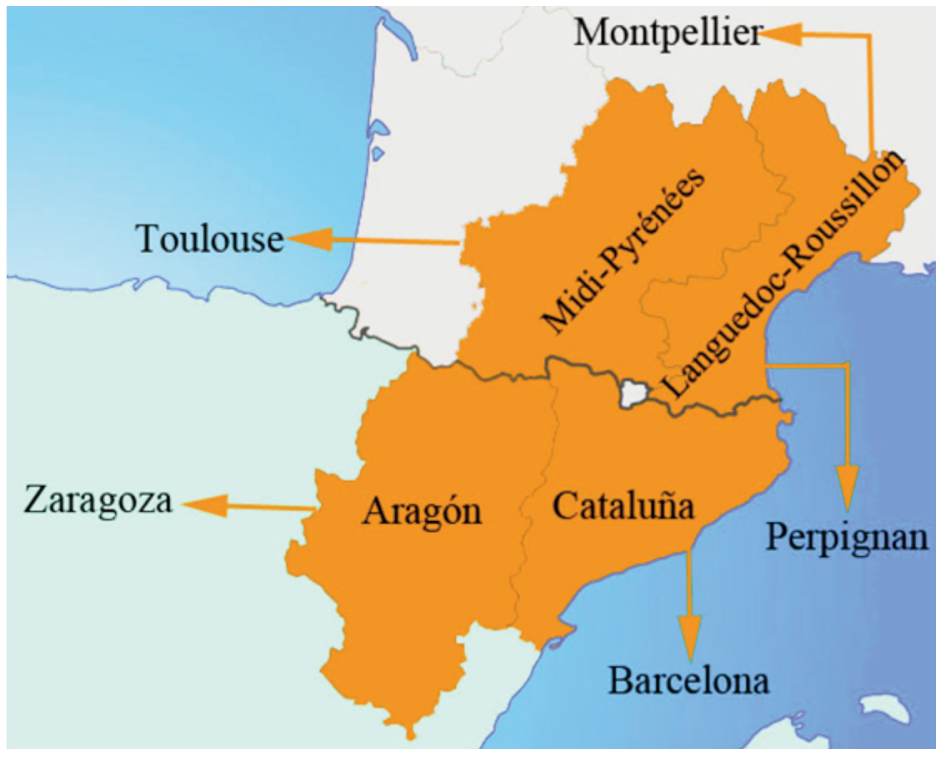

Figura 1. Localización de las cuatro regiones de estudio en España y Francia. Figure 1. The four regions of study in Spain and France.

3. En 1992, la Comisión Europea proporcionó un marco legislativo común (Diario Oficial de la Unión Europea (DOCE) 1992, 2012) para proteger y promocionar alimentos de calidad ligada al origen y/o al sistema de producción. En el caso de la IGP, la producción o la transformación o la elaboración debe realizarse en una zona geográfica determinada (Resano et al., 2012). 


\section{Material y métodos}

\section{La muestra de consumidores}

Los datos analizados en este trabajo se obtuvieron a través de una entrevista personal a una muestra representativa de la población regional en términos del sexo y edad, realizada en las principales ciudades de las cuatro regiones investigadas (Aragón, Cataluña, Midi-Pyrénées y Languedoc-Roussillon) entre septiembre de 2010 y Abril de 2011. Las entrevistas tuvieron lugar en entornos conocidos por los consumidores (centro cívico, asociación de consumidores, lugar de trabajo, centro educativo, etc...) y en grupos reducidos (inferiores a 12 participantes por sesión). La encuesta se dirigió a consumidores habituales de carne de vacuno, que estuviesen involucrados en la compra de alimentos para su consumo en el hogar y fueran mayores de 18 años. La muestra final estaba compuesta por 300 consumidores tanto en Aragón como en Languedoc-Roussillon, 304 en Cataluña y 318 en Midi-Pyrénées, por lo que en total se obtuvieron 1222 encuestas. Los participantes recibieron una pequeña compensación económica para agradecer su colaboración en el estudio 4 .

\section{El cuestionario}

El cuestionario incluía, entre otros aspectos, preguntas sobre los hábitos de consumo y compra de la carne de vacuno, y características socio-demográficas del entrevistado, que se describen con mayor detalle en Sanjuán et al. (2012) y en Sans y Sanjuán-López (2015). Fue necesaria no sólo su traducción a diversos idiomas (castellano, francés y catalán) sino también la adaptación de algunas preguntas a la realidad de cada país/región.

Dos de las preguntas clave del estudio sobre las que se asienta este artículo son: el conocimiento y compra declarados de marcas de vacuno; y la puntuación sobre indicadores de calidad utilizados en el momento de la compra ${ }^{5}$ que se describen a continuación.

Con respecto a la primera cuestión, se presentaba al consumidor un listado de marcas comercializadas en su región, y se le pedía que identificase aquellas que conoce y que ha comprado al menos una vez a lo largo del año previo. La pregunta era dicotómica con las opciones de respuesta "Sí o "No". Dado el elevado número de marcas y distintivos de calidad que existen en cada una de las regiones analizadas, se pueden agrupar en las siguientes categorías: distintivos de calidad que cumplen con la función certificadora de determinado aspecto, en este caso de la carne de vacuno, y está fundamentalmente dirigida a los consumidores (Largo, 2006). Se incluiría al reconocido por la Comisión Europea (IGP) y las marcas de garantía (regionales como puede ser C'alial (anterior C de Calidad) y Label Rouge), y dentro de las denominadas "marcas de empresa" cuya función es indicar el origen o la procedencia empresarial (Largo, 2006), las marcas colectivas cuyo titular es una asociación de productores o de uso individual cuyo titular es un productor (colectiva o individual del productor) y las marcas de uso individual cuyo titular es una cadena de distribución (individual del distribuidor).

Con respecto a la segunda cuestión, se solicitaba a los participantes que señalaran su grado de acuerdo o desacuerdo con que un

4. En la dos regiones españoles se entregaron a los consumidores $10 €$ al contado, mientras que en las dos regiones francesas los consumidores obtuvieron un cheque-regalo para canjear en un establecimiento comercial por valor de $10 €$.

5. Ambas preguntas están disponibles mediante petición a los autores. 
conjunto de características, sobre el lugar de compra, la carne de vacuno, la crianza, el transporte y sacrificio del animal, actúen como indicadores de calidad en el momento de la compra. El nivel de acuerdo se medía en una escala de Likert de 5 puntos equilibrada (1: Totalmente en desacuerdo, 2: En desacuerdo, 3: Ni en desacuerdo ni de acuerdo, 4: De acuerdo, 5: Totalmente de acuerdo).

\section{El análisis estadístico}

En primer lugar, se ha empleado la prueba de Kolmogorov Smirnov para contrastar si la distribución muestral del conocimiento y la compra declarados de la carne de vacuno con marca es o no una normal. En segundo lugar, se ha aplicado el contraste Kruskal WaIlis, que permite averiguar si la información obtenida en las regiones analizadas procede de poblaciones distintas, en cuyo caso habría que realizar el análisis estadístico de los datos obtenidos en cada una de las regiones por separado. Posteriormente, para clasificar a los consumidores en función de su familiaridad con las marcas se ha aplicado el análisis cluster en dos etapas ${ }^{6}$ (two-step clustering). Una vez clasificados los consumidores en los segmentos o clusters definidos, se ha contrastado si existe asociación entre los segmentos obtenidos y el porcentaje que conoce o compra carne con marca mediante el test de Chi-cuadrado, así como entre regiones mediante la prueba $z$ de proporciones. También se han aplicado el estadístico UMann Whitney y el contraste Kruskal Wallis para evaluar la presencia de diferencias pero en este caso respecto a los atributos que utilizan los consumidores como indicadores de calidad en el momento de la compra de carne de vacuno. Finalmente, se investigó si se podía diferenciar el perfil socio-demográfico de los consumidores en función de la pertenencia a un determinado cluster, analizando la asociación entre el segmento y las variables sociodemográficas mediante el estadístico de Chicuadrado, y entre regiones mediante la prueba $z$ de proporciones. Todos los análisis estadísticos se realizaron con IBM SPSS 22.00.

\section{Resultados y discusión}

\section{Conocimiento y compra de carne de vacuno con marca}

En el cuestionario como se ha comentado en la sección anterior se preguntaba a los encuestados que indicaran dentro de un conjunto de marcas de carne de vacuno cuáles conocían y si habían comprado alguna de ellas a lo largo del último año. Los resultados confirman la ausencia de normalidad (Kolmogorov Smirnov: $P<0,05)$, y que la información obtenida en las regiones analizadas procede de poblaciones distintas (Kruskal Wallis: $P<0,05)$.

En la Tabla 1 se observa para el total de la muestra dentro de cada región que prácticamente la totalidad de los participantes conocen y en su mayoría han comprado alguna vez a lo largo del último año carne de vacuno con distintivo de calidad, siendo el porcentaje de compra significativamente distinto (menor) en el caso de las regiones francesas $(60 \%$ en Midi-Pyrénées (M-P) y 55\% en LanguedocRoussillon (L-R) respecto a $70 \%$ en Aragón y $75 \%$ en Cataluña). No obstante, existen algunas diferencias regionales según el tipo de distintivo, dependiendo de si se trata de carne con IGP o de marcas de garantía (regionales o Label Rouge), sobre todo en las re-

6. Esta técnica de segmentación aúna los principios tanto del análisis jerárquico como del no jerárquico iterativo ( $k$-means) y está ganando aceptación en los últimos años en el ámbito de la investigación de mercados (Mooi y Sarstedt, 2011). 


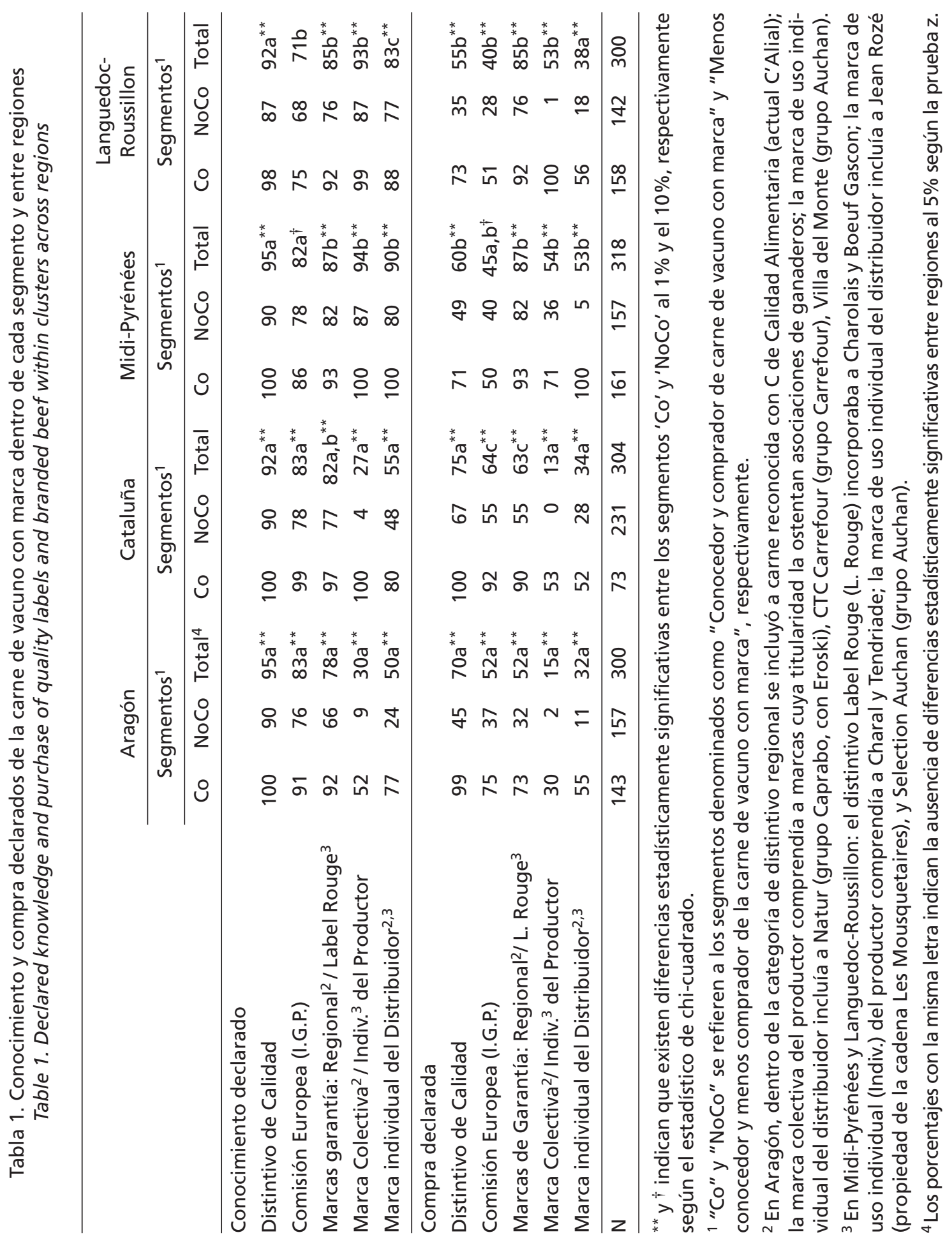


giones francesas. En este sentido, el Label Rouge se conoce, pero fundamentalmente se compra en un porcentaje notablemente superior a la IGP. La mayoría de los participantes franceses compran vacuno con Label Rouge (un $87 \%$ en M-P y un $85 \%$ en L-R), mientras que menos de la mitad lo compran con IGP (un $45 \%$ en $\mathrm{M}-\mathrm{P}$ y un $40 \%$ en L-R), siendo además, este porcentaje significativamente distinto (mayor) del que compra carne con marca de garantía regional en España (63\% en Cataluña y $52 \%$ en Aragón).

Además, el porcentaje de conocimiento y compra del distintivo de calidad es notablemente superior al de las "marcas de empresa" en Aragón y Cataluña. Sin embargo, en M-P y en L-R las diferencias entre estos tipos de marcas son menos apreciables sobre todo en relación al conocimiento sugerido, dado que el de la marca de uso individual del productor y del distribuidor es significativamente superior al de España (entre un 83\% la marca individual del distribuidor en L-R y un $94 \%$ la individual del productor en M-P), mientras que en España se sitúa entre el 30\% (colectiva del productor en Aragón) y un 55\% (individual del distribuidor en Cataluña). Este elevado porcentaje de conocimiento se materializa en una compra declarada para más de la mitad de los participantes franceses, a excepción de L-R con un $38 \%$ en la marca de uso individual del distribuidor.

A pesar de que no hay muchos trabajos que analicen el conocimiento o la compra declarados de carne de vacuno con marca, y que los realizados en España se centran en el análisis de la carne con IGP, los resultados de este estudio se pueden considerar en la línea de la literatura existente. No es de extrañar que el porcentaje de conocimiento declarado en nuestro trabajo sea elevado dado que no se trataba de conocimiento espontáneo sino sugerido. De esta manera, Olaizola et al. (2005) señalaron que el $86 \%$ de los encuestados afirmaban conocer alguno de los distintivos de calidad que se les preguntaba, mientras que este porcentaje disminuía hasta el $64 \%$ cuando no se les sugerían las marcas. En relación con la compra declarada, Sánchez et al. (2001) indicaron que el $75 \%$ de los encuestados en Navarra y País Vasco consumían en su hogar carne de ternera con marca, Olaizola et al. (2005) indicaron en un estudio realizado en Aragón que un $84 \%$ la compraba con relativa frecuencia, y por último Sepúlveda et al. (2008) señalaron que el $73 \%$ de los encuestados en tres regiones (Aragón, Madrid y Castilla León) había comprado con distinta frecuencia carne de ternera con distintivo de calidad.

\section{Segmentación en función del conocimiento y la compra de carne de vacuno con marca}

Se aplicó el análisis cluster de manera separada para cada una de las regiones en función de su conocimiento y compra declaradas de carne con marca. La Tabla 1 presenta los resultados obtenidos dentro de cada segmento además de para el total de la muestra en cada región. El segmento 1 se caracteriza por presentar un mayor nivel de conocimiento y compra de las marcas de carne de vacuno que el segmento 2 en todas las regiones. En consecuencia, se les denomina como "Conocedor y comprador de carne de vacuno con marca" (Co) y "Menos conocedor y menos comprador de carne de vacuno con marca" (NoCo), respectivamente. Los dos segmentos de consumidores presentan tamaños similares, representando aproximadamente la mitad del total de la muestra, a excepción de Cataluña, en la que únicamente un $24 \%$ de la muestra pertenece al segmento "Co".

\section{Calidad percibida en el momento de la compra de carne de vacuno}

En el cuestionario también se preguntaba a los encuestados que señalaran qué atributos indican una mayor calidad en el momento de 
la compra. El estadístico Kruskal Wallis muestra la presencia de diferencias regionales significativas en la mayoría de los atributos que actúan como indicadores de calidad en el momento de la compra. Por lo tanto, después de presentar por orden de importancia en términos medios cuáles son esos atributos en el total de la muestra (Figura 2), se indican las marcas (Tabla 2) y el resto de atributos relevantes (Tabla 3 ) que utilizan como señales de calidad en cada región. No obstante, las Tablas 2 y 3 también incorporan la informa- ción de aquellos atributos en los que no se obtuvieron diferencias significativas en algunas regiones para proporcionar una información más completa y detallada. Un primer resultado a destacar en la Figura 2 es que en promedio, ninguna de las características propuestas se descartan como indicadores de calidad (los valores medios superan el punto medio de la escala). Según esta Figura los atributos más relevantes para inferir la calidad de la carne de vacuno en el momento de la compra son los de confianza, lo que po-

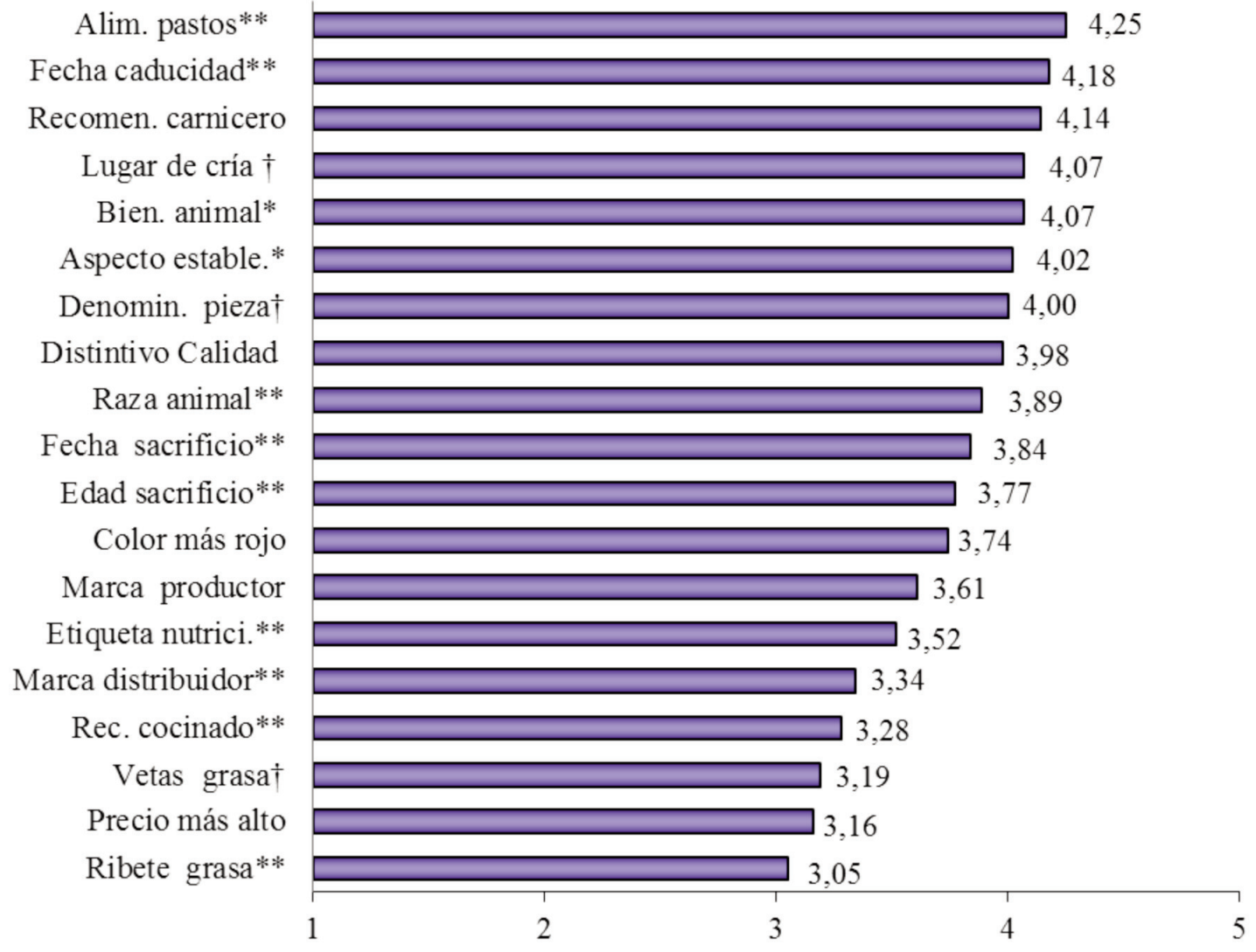

Totalmente (Tot.) en desacuerdo (des.) Des. Ni de acuerdo/des. De acuerdo Tot. de acuerdo

Figura 2. Principales atributos que actúan como indicadores de la calidad en el momento de la compra.

Figure 2. Main attributes indicating quality at the moment of purchase.

**, * $y+$ indican la presencia de diferencias estadísticamente significativas entre las regiones según el estadístico Kruskal Wallis al 1, 5 y 10\%, respectivamente. 





dría estar relacionado con el nivel de experiencia o de familiaridad con el producto (Banovic et al., 2012). En otro estudio realizado con la misma muestra de consumidores (Sanjuán et al., 2012) se ha podido comprobar que los encuestados se consideran a sí mismos más expertos en el consumo y el cocinado de carne de vacuno que en la compra, por lo tanto podría ocurrir que necesiten obtener información en mayor medida de los atributos de confianza. No obstante, también podría ser debido a la mayor preocupación que existe en los últimos años por los temas relacionados con la salubridad y las características saludables de los alimentos como se comentaba en el apartado de la introducción (Espinoza et al., 2015), que se materializa en un mayor interés por el uso de atributos que garanticen esas características.

De manera específica, los resultados indican que la alimentación basada en pastos y la fecha de caducidad actúan como poderosas señales de calidad. Otros atributos relevantes según los encuestados son los relacionados con el lugar de compra, como es el caso de la recomendación del carnicero, y en menor medida el aspecto del establecimiento, así como con aspectos tanto de la crianza del animal (el lugar de cría y el bienestar animal) como de la carne de vacuno (la denominación de la pieza o el corte de la carne). La relevancia de estos atributos se ha confirmado en otros estudios. En este sentido, Bernués et al. (2003b) y Olaizola et al. (2005) señalaron la alimentación del animal y el bienestar animal como los atributos más importantes en la decisión de compra de carne de vacuno, mientras que Bernués et al. (2003a) indicaron que la fecha de caducidad era el atributo más demandado por los consumidores en el momento de la compra. Sánchez et al. (2001) mostraron que la confianza en el carnicero era uno de los atributos más relevantes, mientras que Bernués et al. (2003a) señalaron que el origen y el corte de la carne se encontraban entre los atributos más importantes para determinar la calidad de la carne.
La marca, pero sobre todo el distintivo de calidad es un atributo valorado en el momento de la compra de carne de vacuno por los consumidores según la literatura (Olaizola et al., 2012; Olaizola et al., 2005). En términos medios, el distintivo de calidad es el que más influencia tiene en la percepción de la calidad, siendo a su vez mayor el impacto de la marca del productor que la del distribuidor. Este resultado está en consonancia con los que se han obtenido en otros estudios, como Guerrero et al. (2000), quienes consideraron que a pesar de que la marca del distribuidor está ganando relevancia en las últimas décadas, todavía se percibe como de menor calidad en comparación con la marca del productor.

Por otra parte, Bernués et al. (2003b) u Olaizola et al. (2005), obtuvieron como resultado que la raza se encuentra entre los atributos menos importantes, por lo tanto le asignarían menor relevancia que en nuestro estudio, y argumentan que esto puede ser motivado por la escasa información que recibe el consumidor sobre la raza del animal en el momento de la compra. En nuestro caso, el grado de conocimiento de las distintas razas, y la asociación entre raza y calidad organoléptica por parte de los consumidores, podrían ser objeto de futuros estudios que completarían trabajos previos sobre aceptabilidad sensorial de la carne de razas específicas (Ripoll et al., 2014; Panea et al., 2009).

La fecha y la edad de sacrificio tendrían una importancia media alta, al igual que en Mtimet et al. (2013), mientras que la presencia de un color más rojo y de una etiqueta nutricional se podría considerar como media, siendo la recomendación del modo de cocinado, la existencia de ribete y vetas de grasa infiltrada, o de un precio más alto los menos influyentes. En el caso del precio y la grasa hay que ser cauteloso con el resultado que se obtiene de la puntuación media porque las preferencias de algunos consumidores podrían ser opuestas (Resano et al., 2012), de 


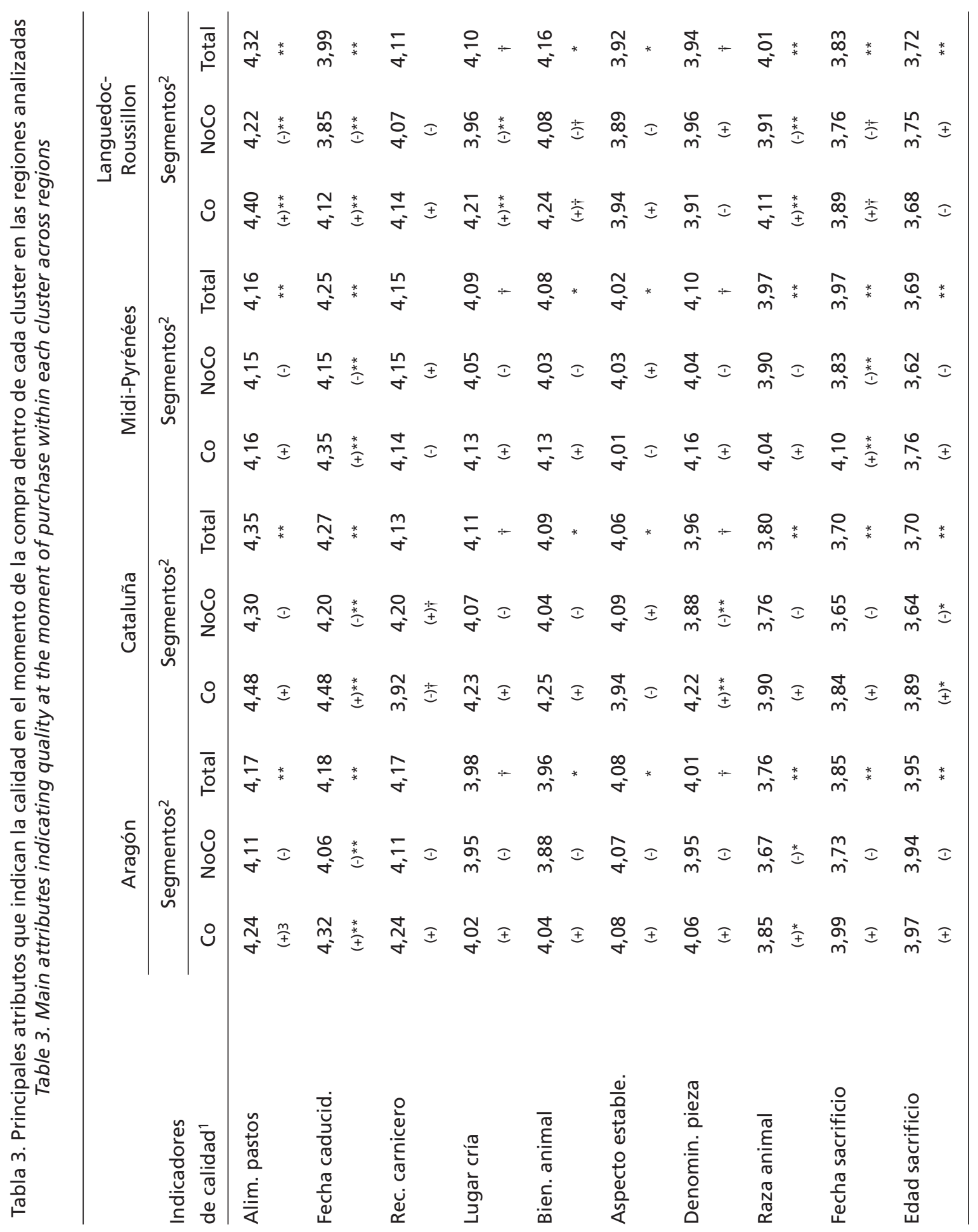




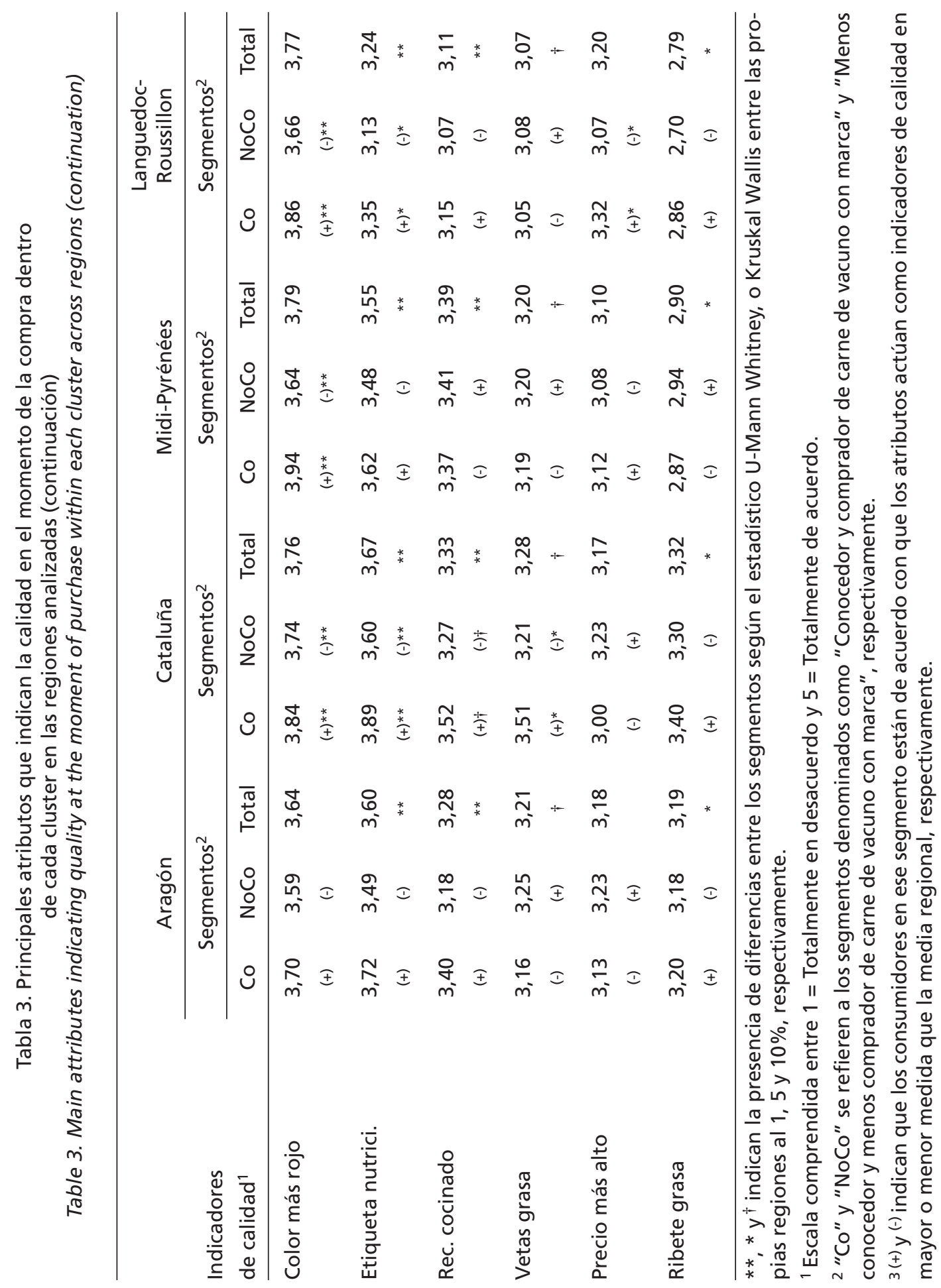


manera que para algunos consumidores el elevado precio o la existencia de cierta cantidad de grasa podrían impedir la compra, mientras que para otros consumidores actuarían como un importante indicador de calidad. De hecho, la cuarta parte de los encuestados muestra su desacuerdo (valores 1totalmente en desacuerdo y 2-en desacuerdo de la escala) con que precio alto, grasa infiltrada o en ribete sean señales de calidad, mientras que otros tantos muestran la opinión opuesta (valores 4-de acuerdo y 5-totalmente de acuerdo de la escala). Sanjuán y Khliji (2016) obtuvieron, mediante un experimento de elección aplicado a la misma muestra de consumidores, que aunque existía una cierta heterogeneidad en las preferencias, los consumidores eran muy sensibles al precio, probablemente por la influencia que la crisis económica ejerce sobre su decisión de compra, y preferían la carne con una cantidad baja o media de grasa. Sánchez et al. (2001) obtuvieron también como resultado que el color, la etiqueta y el precio se encontraban entre los indicadores que menos se asocian con la calidad. La menor importancia concedida a recomendaciones sobre el modo de cocinado también coincide con el resultado de Bernués et al. (2003a) obtenido para los consumidores españoles, lo que puede ser debido a que esta información la puede proporcionar el carnicero cuyo asesoramiento se considera bastante relevante por parte de los encuestados. Estos resultados ponen de manifiesto que hay aspectos no suficientemente comunicados en la actualidad y que, sin embargo podrían ser relevantes de cara a informar al consumidor sobre la calidad de la carne de vacuno. Entre ellos destacan aquellos relacionados con la alimentación del ganado y el bienestar animal. En ausencia de una comunicación verbal por parte del carnicero o de una marca comercial o distintivo de calidad que actúe como garante de estos atributos, se podrían comunicar a través de carteles o etiquetas.
Se obtuvieron diferencias estadísticamente significativas entre regiones en el caso de la utilización de la marca del distribuidor $(P<0,05)$, y en menor medida del productor $(P<0,15)$, sin que se obtuvieran diferencias en el del distintivo de calidad, como se muestra en la Tabla 2. A pesar de ello, y considerando los valores medios en lo que respecta a la marca del productor, Cataluña sería la región donde este tipo de marca actúa como un indicador de calidad más poderoso con una puntuación media de 3,67. En relación con la marca del distribuidor, Cataluña y Midi-Pyrénées son las regiones donde más (con una media de $3,53)$ y donde menos $(3,13)$ relevancia tendría la marca de distribuidor como señal de calidad, respectivamente. De estos resultados se deriva que Cataluña se podría considerar como la región donde los participantes en el estudio emplean en mayor medida la carne de vacuno con marca para inferir la calidad en el momento de la compra.

Sin embargo, dada la dificultad de evaluar la calidad de la carne de vacuno en el momento de la compra, otros atributos pueden actuar como indicadores suplementarios de calidad relevantes, e incluso en mayor medida que la marca, como se observa en la Tabla 3. En particular, Cataluña sería la región donde más se valoran los atributos que para el conjunto de la muestra se observan como más relevantes (los relacionados con la crianza del animal, su transporte o el lugar de compra), en su mayoría conjuntamente con Languedoc-Roussillon, siendo ésta última región a su vez donde menos se valoran los que actúan también como indicadores de calidad en menor medida para el total de la muestra (aquellos relacionados con algunos de los aspectos de la carne como son la presencia de grasa, la recomendación del modo de cocinado y la etiqueta nutricional).

Los resultados revelan la presencia de diferencias interregionales. En particular, Cataluña presenta un comportamiento específico 
en la utilización de marcas, fundamentalmente la marca colectiva del productor y de uso individual del distribuidor, y de otros atributos que son incluso más relevantes que para el conjunto de encuestados para inferir la calidad en el momento de la compra.

\section{Calidad percibida en función de la familiaridad con la carne de vacuno con marca}

La aplicación del estadístico de la U-Mann Whitney demuestra la presencia de diferencias estadísticamente significativas entre segmentos, aunque no en todas las regiones. Según la información presentada en la Tabla 2 el primer segmento que se define como más "Conocedor y comprador de carne de vacuno con marca" es el que está más de acuerdo con que la presencia de una marca actúa como un indicador de la calidad del producto, a pesar de que las diferencias en el caso del distintivo de calidad entre segmentos no sean significativas en Aragón y en Midi-Pyrénées, y en Cataluña sea escasamente significativo $(P<0,15)$. Este resultado confirma la tesis defendida por Banovic et al. (2012), quienes señalan que la influencia que ejercen los indicadores de calidad sobre las expectativas de los consumidores depende de la familiaridad con esos indicadores. Se ha comprobado por tanto que existe un cierto grado de consistencia entre el nivel de familiaridad de los consumidores con las marcas y su utilización como indicadores de calidad.

Además, en términos generales, los atributos suplementarios tienen un impacto mayor para aquellos consumidores que están más familiarizados con la carne con marca. Sánchez et al. (2001) también obtuvieron como resultado de su investigación que los compradores de carne de ternera con distintivo de calidad asignaron mayores puntuaciones que los compradores de carne sin esta marca a todos los atributos que se consideraban relevantes en la decisión de compra, probablemente esto sea debido a su mayor implicación en la compra de carne de vacuno. Asimismo, Sans y Sanjuán-López (2015) demostraron con la misma muestra de consumidores que en este estudio que aquellos consumidores que compran más carne de vacuno con distintivo de calidad, también confían más en la utilización de la certificación de bienestar animal y están dispuestos a pagar más por ella en el momento de la compra.

\section{Perfil de la muestra de consumidores}

En primer lugar, los resultados del test de Chi-cuadrado muestran la ausencia de diferencias significativas entre la muestra y la población regional en términos de sexo y edad. Posteriormente, y después de agrupar a los participantes en los dos segmentos anteriormente definidos en función de su nivel de familiaridad con las marcas de carne de vacuno se ha contrastado si existe asociación entre los segmentos y las características sociodemográficas y de hábitos de consumo de los encuestados mediante el test de Chi-cuadrado (Tabla 4), así como diferencias interregionales considerando la prueba z. De esta manera, el segmento "Conocedor y comprador de carne de vacuno con marca" se diferencia del "Menos conocedor y menos comprador de carne de vacuno con marca" por estar compuesto en mayor proporción por consumidores con edad media (un $60 \%$ de los que se encuentran entre 35 y 65 años pertenecen al segmento conocedor respecto a un $46 \%$ del segundo segmento en Aragón $(P<0,10)$, y en Midi-Pyrénées un $57 \%$ respecto a un $49 \%(P<0,10))$, siendo estos consumidores por lo tanto los que confieren mayor relevancia a la marca o al distintivo de calidad como información determinante de la compra de carne de vacuno. Este resultado está en concordancia con el estudio realizado por Sepúlveda et al. (2008), quienes señalan que los encuestados entre 35 y 64 años son los 


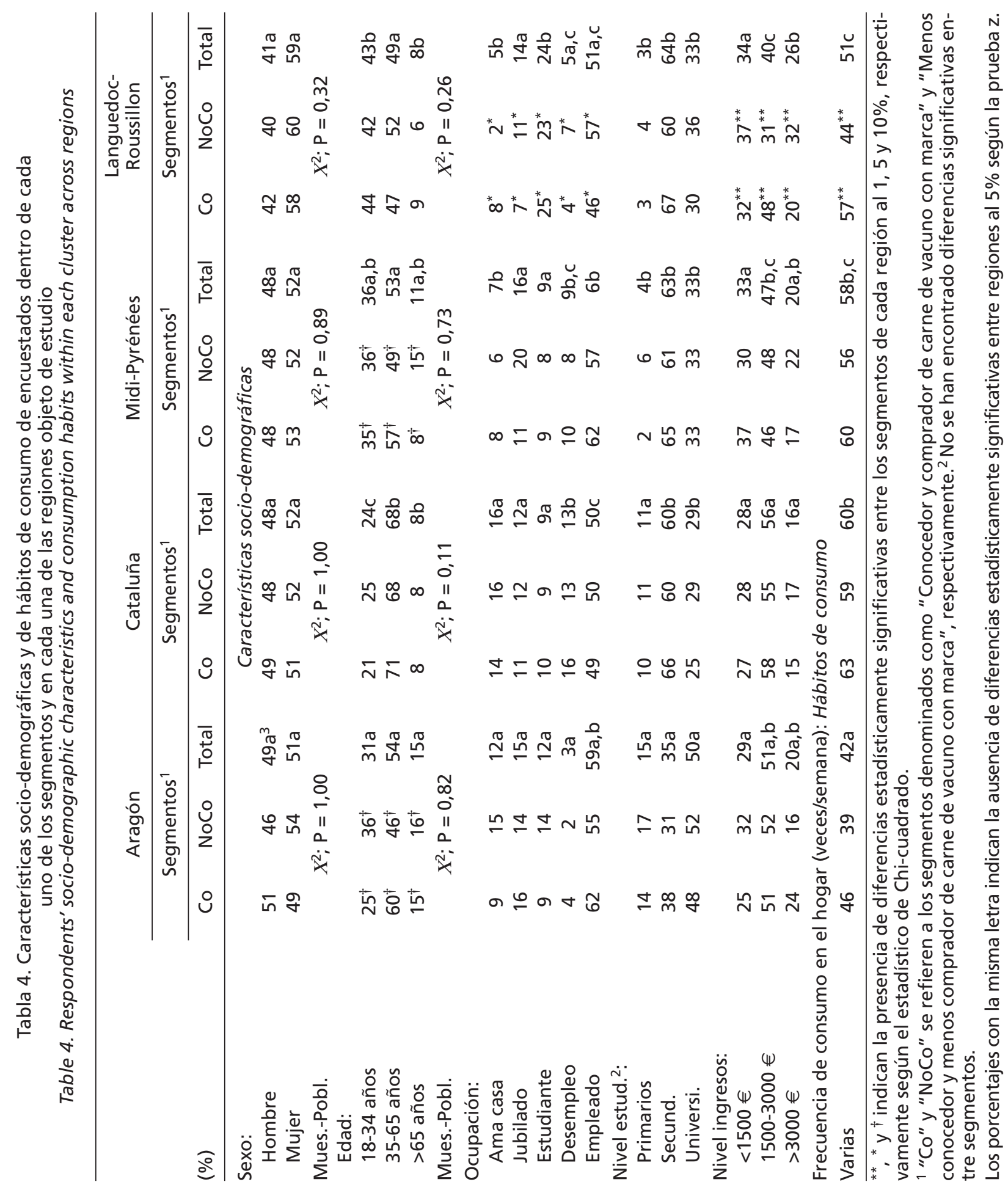


que más compran carne con distintivo de calidad. Sin embargo, no existe un acuerdo en la literatura sobre la influencia de la edad del consumidor sobre su decisión de compra de carne con dicho distintivo. En este sentido, Bernués et al. (2003a, b) afirman que los consumidores jóvenes (menores de 36 años) son los que más compran carne de vacuno con marca, mientras que Martínez et al. (2007) y Sánchez et al. (2001) indican que son aquellos de mayor edad. La renta neta mensual de la unidad familiar del segmento "Conocedor y comprador de carne de vacuno con marca" se encuentra en un mayor porcentaje que en el segundo segmento entre 1500 y $3000 €$ en Languedoc-Roussillon (un $48 \%$ respecto a un $31 \%$ del otro segmento; $\mathrm{P}<0,01)$. Sepúlveda et al. (2008) también señalaron que aquellos consumidores más familiarizados con el distintivo de calidad se caracterizaban a su vez con niveles de renta medio (1500-3000 €) y alto $(3000 €)$, al igual que Olaizola et al. (2005). No se han encontrado diferencias significativas entre segmentos con respecto al sexo, nivel de estudios, y atendiendo a la ocupación de los encuestados, lo más reseñable es que el porcentaje de amas de casa conocedoras y compradoras de carne con marca es mayor ( 8 vs $2 \%$ en Languedoc-Roussillon; $\mathrm{P}<0,05)$ que el perteneciente al segundo segmento, probablemente porque están más involucradas en el proceso de compra para su hogar. En relación a los hábitos de consumo, se ha analizado la frecuencia de consumo en el hogar, como se muestra al final de la Tabla 4. Los resultados indican que aquellos encuestados pertenecientes al primer segmento, consumen a su vez carne de vacuno con mayor asiduidad, aunque estas diferencias sean estadísticamente significativas únicamente en Languedoc- RoussiIlon $(\mathrm{P}<0,01)$. Por lo tanto, se podría asumir la existencia de una cierta asociación entre la familiaridad con la marca y con el propio producto. Además de ello, existen algunas diferencias interregionales $(P<0,05)$ para el to- tal de la muestra en todas las características socio-demográficas a excepción del sexo del encuestado, así como en la frecuencia de consumo. Los diferencias más apreciables se observan en Cataluña con un menor porcentaje de consumidores jóvenes (24\%, situándose en las demás regiones entre un 30 y un $42 \%)$, un mayor porcentaje con edad comprendida entre 35 y 65 años (68\% respecto a aproximadamente la mitad de los participantes en las otras regiones) y un mayor porcentaje de encuestados que consumen varias veces a la semana (rondando el $60 \%$ al igual que en Midi-Pyrénées, respecto a un 42 y un $51 \%$ ), y en Aragón con el menor porcentaje de consumidores con estudios secundarios (35\% respecto a entorno a un $60 \%$ en el resto), y a su vez el mayor de estudios superiores, con la mitad de participantes, rondando el $30 \%$ en el resto de regiones.

\section{Conclusiones}

Este estudio se ha realizado en dos regiones españolas (Aragón y Cataluña) y otras dos francesas (Midi-Pyrénées y Languedoc-Roussillon) situadas a ambos lados de los Pirineos. La novedad del trabajo radica en el análisis de la influencia que ejerce la familiaridad con la carne de vacuno con marca desde tres puntos de vista, que hasta el momento no se han empleado en la literatura académica, o que se han aplicado centrándose únicamente en el estudio de la carne con distintivo de calidad. En primer lugar, el análisis pormenorizado del conocimiento y compra declarados distinguiendo por tipos de marcas; en segundo lugar su impacto sobre la utilización de señales de calidad en el momento de la compra, y por último, la adopción de un enfoque interregional, que permite captar en mayor medida la posible heterogeneidad existente en las áreas geográficas que son objeto de estudio. La investigación se ha llevado 
a cabo fundamentalmente mediante la aplicación de una segmentación en dos etapas, pruebas no paramétricas y de medidas de asociación bivariantes.

Los atributos más relevantes como señales de calidad en el momento de la compra serían los relacionados con la crianza del animal, la fecha de caducidad, los aspectos relacionados con el lugar de compra, y la denominación de la pieza. La marca, pero fundamentalmente el distintivo de calidad sería bastante valorado por los consumidores. La raza, las características del sacrificio, la presencia de un color más rojo o de una etiqueta nutricional tendrían una importancia media. La recomendación del modo de cocinado, o de un precio más alto, y los aspectos relacionados con la grasa serían los indicadores relativamente menos influyentes. Los resultados también indican la presencia de diferencias significativas no sólo entre países sino también entre regiones dentro del mismo país. Este hecho corrobora la importancia de estudiar las diferencias interregionales existentes, mostrando Cataluña un cierto perfil distintivo.

Mediante el análisis cluster aplicado al nivel de conocimiento y compra declarados de carne de vacuno, se han obtenido dos segmentos de consumidores: el primero denominado como más "Conocedor y comprador de carne de vacuno con marca", mientras que el segundo se denomina "Menos conocedor y menos comprador de carne de vacuno con marca", existiendo un cierto grado de consistencia entre el nivel de conocimiento y compra de la carne con marca y la influencia que ejerce al evaluar la calidad del producto. En relación con las características socio-demográficas, se puede mencionar que el segmento más familiarizado con la carne con marca está formado en mayor medida por personas de edad intermedia y nivel de ingresos medios, y no se han encontrado diferencias significativas respecto al sexo del encuestado ni su nivel de estudios. Además, los integrantes de este segmento consumen carne de vacuno con mayor frecuencia, lo que se podría interpretar como que también están más familiarizados con el producto, existiendo por tanto una cierta asociación entre ambos tipos de familiaridad.

Por último, los resultados obtenidos ponen de manifiesto que los productores de carne con marca que vayan a desarrollar una campaña de marketing deberían dirigirla al segmento específico de consumidores que sea potencialmente más receptivo hacia ese producto, considerando las peculiaridades regionales. En este caso, estaría compuesto por consumidores de edad media y nivel de ingresos medios, siendo Cataluña la región más propicia para afianzar el papel que juegan las marcas de carne de vacuno como señales de calidad en el momento de la compra. No obstante, aumentar el reconocimiento de las marcas de carne de vacuno y fomentar la lealtad del consumidor hacia este tipo de atributos podría ser una estrategia efectiva en todos los mercados fronterizos.

\section{Agradecimientos}

Este estudio ha sido financiado con fondos FEDER de la UE, Programa Operativo de Cooperación España Francia Andorra, 2007-2012 (POCTEFA), Proyecto OTRAC. Los autores agradecen a la coordinadora del proyecto (Pilar Santolaria) la ayuda proporcionada y al resto de investigadores su colaboración en la realización de las encuestas a consumidores (Gabriela Zeballos, Ana Guerrero, Pierre Sans, Nuria Panella-Riera, Saoussan Khliji, Maria Angels Oliver, Mari Mar Campo y Carlos Sañudo). 


\section{Bibliografía}

Banovic M, Grunert K, Barreira M M, Fontes M A (2010). Beef quality perception at the point of purchase. A study from Portugal. Food Quality and Preference 20: 335-342.

Banovic M, Fontes M A, Barreira MM, Grunert K (2012). Impact of product familiarity on beef quality perception. Agribusiness 28(2): 157-172.

Bernués A, Olaizola A, Corcoran K (2003a). LabeIling information demanded by European consumers and relationships with purchasing motives, quality and safety of meat. Meat Science 65: 1095-1106.

Bernués A, Olaizola A, Corcoran K (2003b). Extrinsic attributes of red meat as indicators of quality in Europe: an application for market segmentation. Food Quality and Preference 14: 265-276.

BEUC (2015). Close-up on the meat we eat. Consumers want honest labels. The European Consumer Organization. Disponible en:

http://www.beuc.eu/publications/Close-up_ on_the_meat_we_eat_Consumers_want_honest_labels.pdf (acceso abril 2016).

Bredahl L (2003). Cue utilisation and quality perception with regard to branded beef. Food Quality and Preference 15: 65-75.

Brunsø K, Fjord TA, Grunert KG (2002). Consumers' food choice and quality perception. Aarhus School of Business, Aarhus, Denmark. MAPP Working Paper No. 77.

Compés R (2002). Atributos de confianza, normas y certificación. Comparación de estándares para hortalizas. Economía Agraria y de los Recursos Naturales 2(1): 115-130.

Darby MR, Karni E (1973). Free Competition and the Optimal Amount of Fraud. Journal of Law and Economics 16: 67-88.

DOCE (1992). Reglamento del Consejo Europeo 2081/92 (RCE) relativos a la protección de las indicaciones geográficas y de las denominaciones de origen de los productos agrícolas y alimenticios. Diario Oficial de la Unión Europea núm. L. 208/1, de 24 de julio de 1992.
DOCE (2012). Reglamento del Consejo Europeo $1151 / 2012$ (RCE) sobre los regímenes de calidad de los productos agrícolas y alimenticios. Diario Oficial de la Unión Europea núm. L. 343/1, de 14 de diciembre de 2012.

Espinoza T, Mesa FR, Valencia E, Quevedo R (2015). Tipos de fraudes en carnes y productos cárnicos: una revisión. Scientia Agropecuaria 6(3): 223-233.

Grunert KG, Bredahl L, Brunsø K (2004). Consumer perception of meat quality and implications for product development in the meat sector-a review. Meat Science 66: 259-272.

Guerrero L, Colomer Y, Guárdia M D, Xicola J, Clotet $\mathrm{R}$ (2000). Consumer attitude towards store brands. Food Quality and Preference 11: 387-395.

Kotler P, Armstrong G, Saunders J, Wong V (2006). Principles of Marketing. Ed. Pearson Prentice Hall, Europa.

Lancaster KJ (1966). A new approach to consumer theory. Journal of Political Economy 74: 132-157.

Largo R (2006). Las marcas colectivas y las marcas de garantía, 2a Ed. Thomson Civitas, España. $350 \mathrm{pp}$.

Martínez S, Hanagriff R, Lau M, Harris M (2007). Determining the factors affecting demand for branded beef: Applying a logit model to 2004 Neilson home-scan data. Journal of Business \& Economics Research 11(5): 95-102.

Mittal V, Kamakura W A, Govind R (2004). Geographic patterns in customer service and satisfaction: An empirical investigation. Journal of Marketing 68: 48-62.

Mooi E, Sarstedt M (2011). A concise guide to market research. The process, data, and methods using SPSS statistics, 1st Ed. Springer, Nueva York, EE.UU. 306 pp.

Mtimet N, Aroua H, Slimene A (2013). El comportamiento de los consumidores tunecinos de carne de ternera. En: Marketing agroalimentario: Aplicaciones metodológicas y estudios de caso en el contexto global (Ed. EspejelBlanco J, Camarena-Gómez DMJ, Salgado-Beltrán L), pp. 49-60. Pearson, México. 
Nelson P (1970). Information and consumer behaviour. Journal of Political Economy 78: 311329.

Olaizola A, Whebi Z, Manrique E. (2005). Quality perception and consumer attitudes to «specific quality beef» in Aragón, Spain. Spanish Journal of Agricultural Research 3(4): 418-428.

Olaizola A, Bernués A, Blasco I, Sanz A (2012). Perspectivas de una carne de calidad diferenciada: análisis exploratorio para la carne de vacuno "Serrana de Teruel". ITEA: Información Técnica Económica Agraria 108 (4): 1-17.

Oliver R (1993). Cognitive, affective, and attribute bases of the satisfaction response. Journal of Consumer Research 20: 418-430.

Panea B, Casasús I, Blanco M, Joy M (2009). The use of correspondence analysis in the study of beef quality: a case study on Parda de Montaña breed. Spanish Journal of Agricultural Research 7(4): 876-885.

Resano $\mathrm{H}$, Sanjuán A I, Sans $\mathrm{P}$, Panella-Riera N, Campo M M, Khliji S, Oliver M A, Sañudo C, Santolaria P (2011). The role of brands for consumers across the Pyrenees. 57th International Congress of Meat Science and Technology, 7-12 agosto 2011, Gante, Bélgica.

Resano H, Sanjuán Al, Albisu LM (2012). Consumers' response to the EU Quality policy allowing for heterogeneous preferences. Food Policy 37: 355-365.

Ripoll G, Blanco M, Albertí P, Panea B, Joy M, Casasús I (2014). Effect of two Spanish breeds and diet on beef quality including consumer preferences. Journal of Science of Food and Agriculture 94(5): 983-992.

Sánchez M, Sanjuán A l, AkI G (2001). El distintivo de calidad como indicador de seguridad ali- menticia en carne de vacuno y cordero. Economía Agraria y Recursos Naturales 1(1): 77-94.

Sánchez M, Galera MC, Segovia C, Marin GM (2007). Estrategias y acciones de innovación en las empresas agrarias, Ed. Universidad de Almería, España. 288 pp.

Sanjuán Al, Resano H, Zeballos G, Sans P, PanellaRiera N, Campo MM, Khliji S, Guerrero A, Oliver MA, Sañudo C, Santolaria P (2012). Consumers' willingness to pay for beef direct sales. A regional comparison across the Pyrenees. Appetite 58: 1118-1127.

Sanjuán Al, Khliji S (2016). Urban consumers response to the EU mountain labelling: an empirical application in Southern Europe. New Medit 15(1): 72-80.

Sans P, de Fontguyon G, Giraud G (2008). Valuebased labels for fresh beef: an overview of French consumer behaviour in a BSE crises context. International Journal of Consumer Studies 32: 407-413.

Sans P, Sanjuán-López A I (2015). Beef animal welfare, attitudes and Willingness to Pay: A regional comparison across the Pyrenees. Spanish Journal of Agricultural Research 13(3): e0105.

Sepúlveda W, Maza MT, Mantecón AR (2008). Factors that affect and motivate the purchase of quality-labelled beef in Spain. Meat Science 80: 1282-1289.

Thelen S, Ford J B, Honeycutt, E D (2006). The impact of regional affiliation on consumer perceptions of relationships among behavioral constructs. Journal of Business Research 59: 965-973.

(Aceptado para publicación el 22 de septiembre de 2016) 